\title{
Time-dependent behavior of linear polarization in unresolved photospheres, with applications for the Hanle effect
}

\author{
R. Ignace ${ }^{1}$, K. T. Hole ${ }^{1}$, J. P. Cassinelli ${ }^{2}$, and G. D. Henson ${ }^{1}$ \\ 1 Department of Physics \& Astronomy, East Tennessee State University, Johnson City, TN, 37614, USA \\ e-mail: ignace@etsu.edu \\ 2 Department of Astronomy, 2535 Sterling Hall, University of Wisconsin, Madison, WI, 53706-1582, USA
}

Received 9 December 2010 / Accepted 18 March 2011

\begin{abstract}
Aims. This paper extends previous studies in modeling time varying linear polarization due to axisymmetric magnetic fields in rotating stars. We use the Hanle effect to predict variations in net line polarization, and use geometric arguments to generalize these results to linear polarization due to other mechanisms.

Methods. Building on the work of Lopez Ariste et al. (2011, A\&A, 527, A120), we use simple analytic models of rotating stars that are symmetric except for an axisymmetric magnetic field to predict the polarization lightcurve due to the Hanle effect. We highlight the effects for the variable line polarization as a function of viewing inclination and field axis obliquity. Finally, we use geometric arguments to generalize our results to linear polarization from the weak transverse Zeeman effect.

Results. We derive analytic expressions to demonstrate that the variable polarization lightcurve for an oblique magnetic rotator is symmetric. This holds for any axisymmetric field distribution and arbitrary viewing inclination to the rotation axis.

Conclusions. For the situation under consideration, the amplitude of the polarization variation is set by the Hanle effect, but the shape of the variation in polarization with phase depends largely on geometrical projection effects. Our work generalizes the applicability of results described in Lopez Ariste et al., inasmuch as the assumptions of a spherical star and an axisymmetric field are true, and provides a strategy for separating the effects of perspective from the Hanle effect itself for interpreting polarimetric lightcurves.
\end{abstract}

Key words. polarization - techniques: polarimetric - stars: magnetic field - stars: rotation - stars: solar-type

\section{Introduction}

Polarization is a powerful tool in understanding diverse astrophysical phenomena. Net polarization in the continuum and spectral lines can measure spatial, kinematic and compositional structure that could not otherwise be detected in unresolved sources. It can also be used as a diagnostic of magnetic fields in both resolved and unresolved sources. Continuum polarization can be produced by Thomson scattering; in lines it can be created via the Hanle and tranverse- and longitudinal-Zeeman effects. In this paper we focus on the Hanle effect, but our results can also apply to other magnetically-induced linear polarization mechanisms.

The Hanle effect is a weak field case of the Zeeman effect with consequence for resonance line scattering polarization in the presence of a magnetic field (e.g., Moruzzi \& Strumia 1991; Stenflo 1994; Landi Degl' Innocenti \& Landolfi 2004). The Hanle effect describes the influence of the field for the linear polarization of line scattering; unlike the Zeeman effect, the Hanle effect does not generate circularly polarized emissions.

The Hanle effect has proven to have diagnostic value in a number of applications to solar physics. Examples from the recent literature include coronal magnetic field (e.g., Derouich et al. 2010), turbulent magnetic fields (e.g., Frisch et al. 2009; Rachkovskii 2009; Kleint et al. 2010), chromospheric magnetic fields (e.g., Faurobert et al. 2009), and prominences (e.g., Merenda et al. 2006), to name only a few. There have also been efforts to develop diagnostics based on the Hanle effect for molecular lines (e.g., Berdyugina \& Fluri 2004;
Shapiro et al. 2007). Many of these diagnostics have been developed to interpret the so-called "Second Solar Spectrum" (Stenflo \& Keller 1997). There has even been a consideration of the Hanle effect for the magnetic field of Jupiter (Ben-Jaffel et al. 2005).

For stars other than the Sun, considerations of the Hanle effect have so far been restricted to theoretical calculations, such as simplified considerations in stellar wind lines (e.g., Ignace et al. 2004), circumstellar disks (Yan \& Lazarian 2008; Ignace 2010), and maser sources (Asenio Ramos et al. 2005). So far, no definitive detections of the Hanle effect in the stellar context have been reported.

A new consideration of the Hanle effect in photospheric lines of unresolved stars has been proposed by Lopez Ariste et al. (2011). Our paper makes a contribution to these new and interesting results by extending them to a more general case through a consideration of perspective effects for oblique magnetic rotators. We demonstrate that for a given field topology and field strength, aspects of the variable polarization due to geometry can be disentangled from the mechanism of the Hanle effect and generalized to apply to any net polarization due to a bipolar magnetic field. Section 2 presents the background for our models: Sect. 2.1 describes polarization conventions and gives an overview of the nature of the Hanle effect. Adopted geometry and assumptions are defined in Sect. 2.2, and solutions for perspective effects for polarimetric lightcurves are given in Sect. 2.3. There are three parts to the discussion: (a) a consideration of an edge-on (or equator-on) rotating star as discussed by Lopez Ariste et al. (2011); (b) a generalization to arbitrary 
viewing inclination of the stellar rotation axis; and (c) a review of a special case in which the dependence of amplitude on the Hanle effect is a known function of the inclination between the field and observer axes. Concluding remarks are given in Sect. 3.

\section{The Hanle effect in unresolved photospheres}

\subsection{Polarization and the Hanle effect}

Polarization is measured in terms of the four Stokes vector components, $I, Q, U$ and $V$. Here $I$ is total intensity; $Q$ and $U$ measure the intensity of linearly polarized light relative to two axes offset by a rotation of $45^{\circ}$; and $V$ is a measure of the circularly polarized light - in our case zero. For convenience we introduce normalized parameters $q=Q / I$ and $u=U / I$.

Measured Stokes parameters are defined with respect to one orientation in the sky - by convention, $q$ is measured along the North-South axis. Of course, some arbitrary source in the sky will not normally have a favorable orientation with respect to the observer convention. In addition to measuring polarized flux in the Stokes 4 parameter system, it is useful to have a relative measure of the polarization that is independent of the observer system.

With a focus on the linear polarization, the observerdependent $q$ and $u$ values can be recast in terms of a polarization magnitude and orientation on the sky. These are $p$, the fraction (or percentage) of polarized light, and $\psi$, the position angle. They are defined as

$p=\sqrt{q^{2}+u^{2}}$

and

$\tan 2 \psi=\frac{U}{Q}=\frac{u}{q}$

These definitions are crucial to arguments that will appear in the following sections. Measures of polarization using $(q, u)$ and $(p, \psi)$ are operationally analogous to Cartesian and polar coordinates. In this respect $p$ acts like a radius. A rotation of the coordinate frame to a new system affects the $(q, u)$ measures, but not $p$.

The Hanle effect can produce a net polarization in the line in the following way. First consider a spherically symmetric star that has no magnetic field. The polarization profile is centrosymmetric, so that as an unresolved source, the star has net zero polarization. The radial profile of the polarization is zero at the projected center of the star, and has a maximum value denoted by $p_{0}$ at the stellar limb with an orientation tangent to the limb. The value of $p_{0}$ can vary from one line to the next. Indeed, even the shape of the run of polarization from center to limb can differ between lines, but this will not affect our conclusions.

The presence of a magnetic field breaks the symmetry across the projected stellar disk leading to a net polarization in the line. A semi-classical description explains the Hanle effect in terms of a damped harmonic oscillator. The magnetic field serves to precess the orientation of the oscillator, leading to a change in polarization position angle and amplitude relative to the zerofield case (Hanle 1924). As a result, a net polarization in an unresolved line from an unresolved photosphere can be produced that is a function of the field geometry, field strength, and the line transition under consideration. The analysis that follows emphasizes geometrical considerations and symmetries for the case of polarized lines from oblique magnetic rotators.

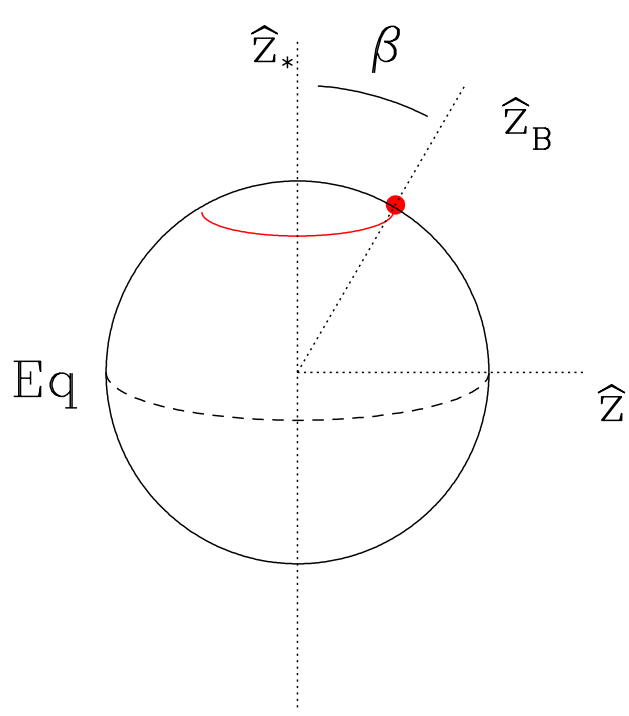

Fig. 1. Illustration of the relation between the rotation, magnetic, and observer axes $\left(z_{*}, z_{B}\right.$, and $z$ respectively) as seen equator-on. The dashed line is the equator and the red arc is the path followed by the magnetic pole as the star rotates. The red dot is the intersection of the magnetic axis with the stellar surface (compare with Fig. 2). In this figure the magnetic pole is currently at longitude of zero (i.e., $\phi=0^{\circ}$ ).

\subsection{Conventions and assumptions}

To investigate the observational implications of magnetic-fieldinduced polarization in rotating stars, we make a number of reasonable simplifying assumptions. (See Fig. 1 for the definition of our geometry.)

- Except for the field distribution, the star is otherwise spherically symmetric.

- The star is taken to rotate as a solid body. The observer is located in direction $\hat{z}$ from the center of the star. The rotation axis is in direction $\hat{z}_{*}$. The viewing inclination $i_{0}$ is defined by

$\hat{z} \cdot \hat{z}_{*}=\cos i_{0}$.

- The magnetic field is dipolar and axisymmetric. Its symmetry axis is in direction $\hat{z}_{B}$. The obliquity angle $\beta$ between the field axis and the rotation axis is defined by

$\hat{z}_{*} \cdot \hat{z}_{B}=\cos \beta$

- We adopt the definition of stellar longitude introduced by Lopez Ariste et al., using the angle $\phi$ to represent the longitude of the field axis on the stellar surface. (The location of this axis is of course simply a point.) Zero longitude $\left(\phi=0^{\circ}\right)$ means that the magnetic north pole lies in front of the star along the bisector (or "Prime Meridian") of the observer's projected stellar disk in the sky.

Figure 2 gives an illustration of the projected stellar disk. The dashed line is the equator; dotted is the bisector line with $\phi=$ $0^{\circ}$. The red dot represents the location of the intersection of the magnetic axis (which passes through the star's center) with the stellar surface. This axis is inclined by the angle $\beta$ from the north pole (top of the circle).

We use this bisector line to define observer axes for measured Stokes parameters $I, Q, U$, and $V$. Circular polarization is not relevant to the discussion, so $V=0$. The angle $\psi$ is the reference orientation angle for the field axis on the stellar surface 


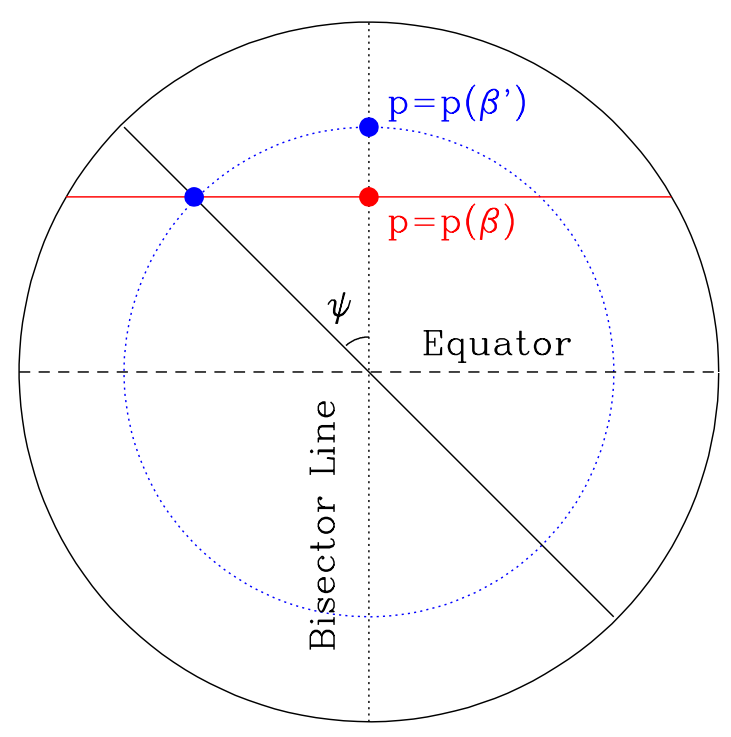

Fig. 2. Illustration of the projected stellar disk for a star seen equator-on to its rotation. The equator is indicated. The "bisector line" defines zero longitude $\left(\phi=0^{\circ}\right)$ on the star. The red line represents the constant colatitude of rotation for the magnetic axis at the point of interception with the stellar surface. The blue dotted circle indicates the locus of points which would have a constant polarization $p$ if the magnetic pole were located on that curve, with the two blue dots as examples.

as measured counterclockwise around the observer axis from the bisector line. Stokes $Q$ and $U$ are defined in terms of the net surface integrated polarized flux arising from the Hanle effect and in reference to this orientation angle. Tranformation of our $Q$ and $U$ values to some other observer system $Q^{\prime}$ and $U^{\prime}$ is easily accomplished by means of a rotation through an angle $2 \psi_{0}$, viz.

$\left(\begin{array}{c}Q^{\prime} \\ U^{\prime}\end{array}\right)=\left(\begin{array}{rr}\cos 2 \psi_{0} & \sin 2 \psi_{0} \\ -\sin 2 \psi_{0} & \cos 2 \psi_{0}\end{array}\right)\left(\begin{array}{c}Q \\ U\end{array}\right)$

The point of going through the exercise of the preceding expression is that net values of $Q$ and $U$ in the line will most easily be evaluated in a reference system defined by the field axis because of the underlying physics, but polarized fluxes will be measured in an observer system (the primed system). The quantity that is preserved in this transformation is the polarization $p=\sqrt{q^{2}+u^{2}}=\sqrt{q^{2}+u^{\prime 2}}$.

We stress that, as in Lopez Ariste et al. (2011), the polarization under consideration is the total line polarization. We are not discussing temporal behavior within resolved lines.

\subsection{Analytic solutions}

We take as given that solutions for $p$ as a function of field strength $B$, obliquity $\beta$, and viewing inclination of the rotation axis $i_{0}$ have already been found for the line transition under consideration, for example from calculations like those given in Lopez Ariste et al. (2011; see their Figs. 1 and 2). That paper presents a lightcurve in their Fig. 3 that shows an asymmetric variable polarization with respect to zero longitude, corresponding to our bisector line with $\phi=0^{\circ}$. We believe, however, that the lightcurve should be mirror symmetric about this reference line, for reasons presented in the next section. Finally, we develop an extension of that conclusion in relation to general axisymmetric field distributions for rotating stars viewed at arbitrary inclinations. We then discuss a particular application that has been presented in Ignace (2001).

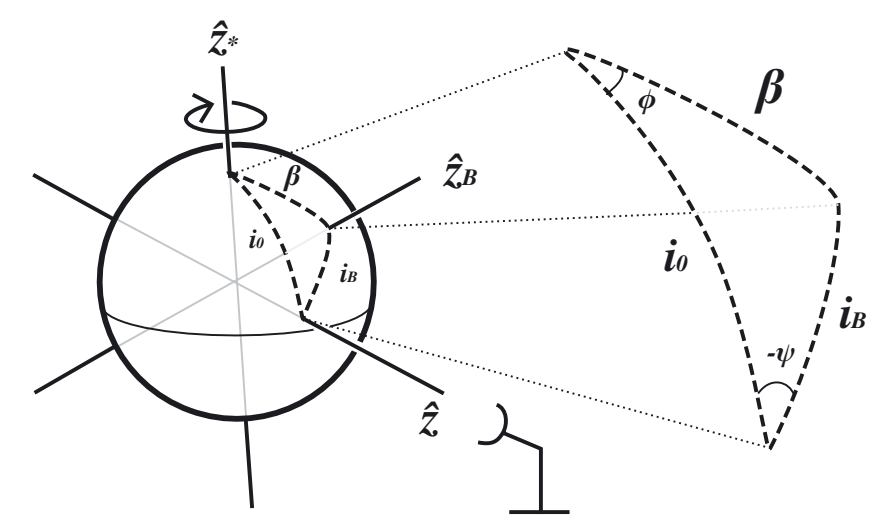

Fig. 3. The three dimensional geometry for the general case of arbitrary inclination of the rotation axis. The close-up shows details of the spherical triangle defined by the three axes in the problem, those of the observer, the star's rotation, and the magnetic field. Symbols are defined in the text. Axes meet at the center of the star, but are not necessarily perpendicular to each other.

\subsubsection{Symmetry of the polarization lightcurve}

Let the rotating star be viewed equator-on, with $i_{0}=90^{\circ}$. Suppose that an ensemble of calculations provide the line polarizations for this case. As the star in Fig. 2 rotates, the red dot would track along the red line. When it passes behind the star, the opposite magnetic pole would appear in the southern hemisphere in a mirror-symmetric way.

The blue dot that lies on the red line is the projected location of the magnetic axis at some time during the rotation. For the observer that dot (the magnetic pole) is at an orientation of $\psi$; in the star system, the dot is located at longitude $\phi$. Recall that the star is spherically symmetric, the field is axisymmetric, and the photodisk is unresolved. This means the total observed polarization from the star must correlate with the location of the magnetic pole and be a periodic function of rotational phase. We refer to this polarization as $p\left(90^{\circ}, \beta, B, \phi\right)$.

An efficient way to think of the polarized lightcurves is to employ the fact that polarization $p$ is invariant with rotation of the observer reference frame. If one were to take the blue dot on the red line and simply rotate it about the observer axis, it would trace out the dotted blue circle shown in Fig. 2. Remember, the bisector line in Fig. 2 is defined in relation to the star's rotation axis and cannot generally be expected to line up with the NorthSouth direction at Earth! Will the failure of these two to line up change the observed polarization? Of course not, because $p$ is invariant under such a rotation.

Therefore, rotating a field of the same topology, strength, and projection as signified by the blue dot on the red line to any other position on the dotted blue circle will not change the value of $p$. The blue circle intersects the bisector line. We see then that the polarization at any rotational longitude (or rotational phase) is equivalent to the polarization value on the bisector line for a different field obliquity $\beta^{\prime}$.

This graphical construction leads to the following relation:

$p\left(90^{\circ}, \beta, B, \phi\right)=p\left(90^{\circ}, \beta^{\prime}, B, 0\right)$.

Simple spherical trigonometry gives

$\sin \beta^{\prime}=\sin \beta \cos \phi$, and
$\tan \psi=-\frac{\tan \phi}{\cos \beta}$. 
As expected, $\beta^{\prime}=\beta$ and $\psi=0^{\circ}$ when the field axis is oriented along zero longitude of $\phi=0^{\circ}$, the bisector line.

In this edge-on scenario, knowing the polarization from models as a function of line transition, field strength, and (axisymmetric) field topology along the bisector line alone allows one to construct polarized lightcurves for oblique magnetic rotators. Variations in $q$ and $u$ will be given by:

$$
q=p\left(90^{\circ}, \beta^{\prime}, B, 0^{\circ}\right) \cos (2 \psi),
$$$$
u=p\left(90^{\circ}, \beta^{\prime}, B, 0^{\circ}\right) \sin (2 \psi) \text {. }
$$

The relations of Eqs. (6)-(8) demonstrate that $\beta^{\prime}$ takes on the same value at $\phi$ as for $-\phi$. Therefore the polarimetric lightcurves must be symmetric about zero longitude.

\subsubsection{Generalization to arbitrary inclinations}

Figure 3 illustrates the spherical geometry in the general case of an arbitrary viewing inclination. The direction of the rotation axis is $\hat{z}_{*}$. The observer is located in the direction of $\hat{z}$ in this figure, and the magnetic axis in the direction of $\hat{z}_{B}$. The angle $i_{B}$ represents the effective viewing inclination of the field axis at any moment. Note that $\phi$ is measured from the plane defined by the observer and rotation axes as measured counterclockwise around $z_{*}$. The observer orientation $\psi$ is similarly defined in relation to the $z$-axis. In the spherical triangle of Fig. $3,-\psi$ appears because the magnetic pole lies clockwise of the reference plane.

For purposes of generalization, the argument of the previous section requires some new considerations. If the rotation axis is not seen equator-on, it might not be the case that the polarization is invariant for the field axis located anywhere around the blue curve. (Note that the pole-on case is trivial, with no variation in polarization.) But consider the following.

- If the field is axisymmetric and top-down symmetric, then $p$ is invariant around the blue circle.

- If the field is axisymmetric, but not top-down symmetric, meaning it is not mirror symmetric about the magnetic equator, then $p$ is not invariant around the blue circle. When the field axis (i.e., the red dot in Fig. 2) is at $\phi<-90^{\circ}$ or $\phi>+90^{\circ}$, the observer sees more of the southern magnetic hemisphere than the northern, which is the opposite of the case when $\phi>-90^{\circ}$ and $\phi<90^{\circ}$. However, it remains the case that the lightcurve is symmetric about zero longitude.

Here a comment regarding the meaning of "top-down symmetric" is needed. The divergence of a magnetic field is zero. The implication for top-down symmetry is that the field topology above and below the magnetic equator is identical except for a vector reversal of the field, meaning $\boldsymbol{B}_{\text {North }}=-\boldsymbol{B}_{\text {South }}$ for the poloidal component of the field.

Technically, this kind of symmetry would matter for the Hanle effect. A sign flip of the vector field does change $Q$ and $U$ qualitatively: the precession of the oscillating charge in the semi-classical description of the Hanle effect now proceeds with opposite handedness. But at the same field strength, the total polarization is not changed. In our "top-down" symmetry, a change in the sign of the vector field does not affect the amplitude of the polarization.

To summarize, the Hanle effect modifies the polarized surface map across a star. That map depends on three things: (a) the field topology, (b) the field strength, and (c) the orientation of the field with respect to the observer. However, the star is not resolved, so a given polarimetric map from a "snapshot" of the star reduces to a single value of $q$ and $u$, or equivalently $p$ and $\psi$. If the field is axisymmetric and top-down symmetric, then for a given field topology and field strength, the value of the net polarization $p$ tracks with the projected location of the field symmetry axis on the star only. This means $p$ is a constant for an axis location on circles of constant projected-disk radius centered on the observer's line-of-sight to the star center.

As a result of these arguments, $p=p\left(\{\hat{B}\}, B, i_{B}\right)$, where $\{\hat{B}\}$ represents the field topology at the location of line formation, where $B$ again is the field strength, and now $i_{B}$ is the instantaneous angle between the observer and the field axis, given by $\cos i_{B}=\hat{z}_{B} \cdot \hat{z}$. Knowing the polarization for all $i_{B}$ amounts to knowing the polarization for a magnetic axis at any location on the projected disk. Schematically, polarimetric lightcurves can be constructed by simply considering how the point of the magnetic pole on the star moves through this grid of known solutions for $p$ as a function of longitude $\phi$.

The time behavior of the polarization is cyclic, symmetric, and governed by how the magnetic pole tracks across the face of the projected stellar disk which is set by the angles $i_{B}$ and $\psi$. Working out the spherical trigonometry based on Fig. 3, solutions for $i_{B}$ and $\psi$ in terms of field axis obliquity, rotation axis inclination, and longitude of the magnetic pole are given by

$$
\begin{aligned}
\cos i_{B} & =\cos \beta \cos i_{0}+\sin \beta \sin i_{0} \cos \phi, \\
\tan \psi & =-\frac{\sin \beta \sin i_{0} \sin \phi}{\cos \beta \sin i_{0}+\sin \beta \cos i_{0} \cos \phi} .
\end{aligned}
$$

The $q$ and $u$ Stokes parameters at any time (or phase) in the lightcurve will now be given by

$q=p\left(\{\hat{B}\}, B, i_{B}\right) \cos (2 \psi)$
$u=p\left(\{\hat{B}\}, B, i_{B}\right) \sin (2 \psi)$

where $i_{B}$ and $\psi$ are implicit functions of the longitude $\phi$.

The top-down symmetry as we have described ensures that $p$ is a constant regardless of which end of the magnetic field axis appears on the projected stellar disk. For certain values of $i_{0}$ and $\beta$, the north magnetic pole can rotate behind the star and be eclipsed. At those moments of ingress and egress, the south magnetic pole will be at egress or ingress, respectively. Although $p$ does not change, $\psi$ tracks with the location of the field axis and thus suffers a $180^{\circ}$ rotation. However, this will produce no observable effect since $q$ and $u$ depend on $2 \psi$, and so are periodic with $180^{\circ}$.

One interesting note is that the ratio of $u / q$ (and thus $\psi$ ) is completely independent of the Hanle effect and derives purely from geometrical considerations of the problem. The ratio is

$\frac{u}{q}=\tan (2 \psi)=\frac{2 \tan \psi}{1-\tan ^{2} \psi}$

and contains information about $i_{0}$ and $\beta$ only. This result thus provides an interesting control in the modeling effort of observations by relegating the influence of the Hanle effect to the shape of the total polarization lightcurve.

\subsubsection{Special case of separable functionality}

Without knowing the exact dependence of the polarization with angle $i_{B}$, it is not possible to evaluate $q$ and $u$ any further than expressed in Eqs. (13)-(14). However, there is at least one case where that dependence is known.

Ignace et al. (1997) considered the Hanle effect for optically thin resonance line scattering in circumstellar media, 
such as winds and disks. For a spherically symmetric wind and an axisymmetric field topology (analogous to the photospheric case considered by Lopez Ariste et al. 2011), Ignace et al. (1997) showed that the net line polarization scales as $p \propto f(\boldsymbol{B}, n) \sin ^{2} i_{B}$. Here $n$ relates to the spherical number density distribution. The function $f$ depends on the density (in effect, the line optical depth), the field strength and topology, and the particular line transition. Ignace (2001) then considered the Hanle effect from oblique magnetic rotators for emission lines that form in a spherically symmetric circumstellar environment.

Although the adoption of spherical symmetry in the circumstellar environment is not a physically realistic scenario, it does serve as a concrete example in which the conclusions of the preceding sections can be illustrated. The factor $\sin ^{2} i_{B}$ is a particular solution for thin lines when treating the star as a point source in terms of illumination and occultation. Under these circumstances, the Hanle effect appears only as a scale parameter that sets the amplitude of the polarization completely isolated from the $i_{B}$-dependence. Thus, polarimetric variations arise strictly from geometrical considerations in $q / f$ and $u / f$, as displayed in Fig. 2 of Ignace (2001).

\section{Conclusions}

We have shown that the polarimetric lightcurves arising from the Hanle effect in photospheric lines of oblique magnetic rotators are mirror symmetric in relation to a stellar-disk "bisector" line. We have additionally described a methodology extending the interesting results presented in Lopez Ariste et al. (2011) for the equator-on case to more general viewing inclinations. We have further shown explicitly that the ratio $u / q$ is independent of the Hanle effect and varies with time purely as a function of perspective variables for the magnetic axis.

In fact the geometric properties that we have employed in relation the lightcurves for Hanle polarization have more general applicability. Fox (1992) showed applicability of this approach to polarizations arising from electron scattering in stellar envelopes. This has been used in simulating variable polarizations from clumped wind flows. Davies et al. (2007) and Li et al. (2009) derive the polarization from an individual electron scattering wind clump and then use the geometric properties emphasized in this paper to "populate" a wind with many clumps to explain observed variable polarizations in Luminous Blue Variable stars and Wolf-Rayet stars.

Moreover, the results should hold for the total linear polarization of lines in which the transverse Zeeman effect is operating. Indeed, Landolfi et al. (1993) demonstrate this explicitly in the "weak" limit of the transverse Zeeman effect. For an oblique magnetic rotator with a dipole field, Eq. (14) and Fig. 2 of Landolfi et al. (1993) are to be compared with (as it happens) Fig. 2 for the special case noted above due to the Hanle effect in Ignace et al. (2001). The two are in fact the same in terms of geometrical and time variable dependence.

The end result is that we find aspects of geometry that decouple from the physical mechanisms that give rise to observed variable polarimetric signals. This is important for distinguishing geometrical effects from source properties. Furthermore in the case of the Hanle effect, a diagnostic approach that evaluates polarizations in different lines will prove important. This is because for a given field strength, different lines have different levels of response to the Hanle effect. Ignace et al. (1997) discussed the power of a multi-line approach for discerning circumstellar magnetic fields from polarized line data. Although they dealt with a steady-state scenario, the advantages that come from a multiline analysis will be useful in deriving Hanle effect solutions for field geometries and strengths in oblique magnetic rotators.

Acknowledgements. The authors are indebted to the referee V. Bommier for a thorough review of this manuscript. We gratefully acknowledge funding for this work provided by the National Science Foundation, grant AST-0807664.

\section{References}

Asenio Ramos, A., Landi Degl'Innocenti, E., \& Trujillo Bueno, J. 2005, ApJ, 625,985

Ben-Jaffel, L., Harris, W., Bommier, V., et al. 2005, Icar, 178, 297

Berdyugina, S. V., \& Fluri, D. M. 2004, A\&A, 417, 775

Davies, B., Vink, J. S., \& Oudmaijer, R. D. 2007, A\&A, 469, 1045

Derouich, M., Auchere, F., Vial, J. C., \& Zhang, M. 2010, A\&A, 511, A7

Faurobert, M., Derouich, M., Bommier, V., \& Arnaud, J. 2009, A\&A, 493, 201

Fox, G. K. 1992, Ap\&SS, 187, 219

Frisch, H., Anusha, L. S., Sampoorna, M., \& Nagendra, K. N. 2009, A\&A, 501, 335

Hanle, W. 1924, Z. Phys., 30, 93

Ignace, R. 2001, in Advanced Solar Polarimetry, ed. M. Sigwarth, ASP Conf. Ser., 236 (San Francisco: ASP), 227

Ignace, R. 2010, ApJ, 725, 1040

Ignace, R., Nordsieck, K. N., \& Cassinelli, J. P. 1997, ApJ, 486, 550

Ignace, R., Nordsieck, K. N., \& Cassinelli, J. P. 2004, ApJ, 609, 1018

Kleint, L., Berdyugina, S. V., Shapiro, A. I., \& Bianda, M. 2010, A\&A, 524, A37

Landi Degl'Innocenti, E., \& Landolfi, M. 2004, Polarization in Spectral Lines (Dordrecht: Kluwer)

Landolfi, M., Landi Degl'Innocenti, E., Landi Degl'Innocenti, M., \& Leroy, J. L. 1993, A\&A, 272, 285

Li, Q.-K., Cassinelli, J. P., Brown, J. C., \& Ignace, R. 2009, RAA, 9, 558

Lopez Ariste, A., Asensio Ramos, A., \& Gonzalez Fernandez, C. 2011, A\&A, 527, A 120

Merenda, L., Trujillo Bueno, J., Landi Degl'Innocenti, E., \& Collados, M. 2006, ApJ, 642, 554

Moruzzi, G., Strumia, F. 1991, The Hanle Effect and Level-Crossing Spectroscopy (New York: Plenum Press)

Rachkovskii, D. N. 2009, Astron. Rep., 53, 153

Shapiro, A. I., Berdyugina, S. V., Fluri, D. M., \& Stenflo, J. O. 2007, A\&A, 475, 349

Stenflo, J. O. 1994, Solar Magnetic Fields (Dordrecht: Kluwer)

Stenflo, J. O., \& Keller, C. U. 1997, A\&A, 321, 927

Yan, H., \& Lazarian, A. 2008, ApJ, 677, 1401 